\title{
Chapter 1 \\ A parallel numerical solution approach for nonlinear parabolic systems arising in air pollution transport problems
}

János Karátson and Balázs Kovács

\subsection{Introduction}

The subject of this paper is the numerical treatment of nonlinear parabolic systems, which arise in various situations in the mathematical modeling of time-dependent reaction-convection-diffusion transport systems, in particular those describing meteorological phenomena and the effect of air pollution $[16,7,8,14,15]$. Such systems normally lead to large-scale and computationally complicated problems, hence it is important to develop efficient and mathematically solid numerical solution algorithms.

We consider systems which contain nonlinear coupling in the reaction terms, further, involving mixed boundary conditions and localized interface conditions as well. That is, in the general case we consider a system of the form

\footnotetext{
J. Karátson

Department of Applied Analysis \& MTA-ELTE NumNet Research Group, ELTE University; Department of Analysis, Technical University; Budapest, Hungary, e-mail: karatson@cs.elte.hu

B. Kovács

MTA-ELTE NumNet Research Group, ELTE University, Budapest, Hungary e-mail: koboaet@cs.elte.hu
} 


$$
\left.\begin{array}{rl}
\frac{\partial u_{i}}{\partial t}-\operatorname{div}\left(K_{i} \nabla u_{i}\right)+\mathbf{b}_{i} \cdot \nabla u_{i}+R_{i}\left(x, u_{1}, \ldots, u_{\ell}\right) & =f_{i} \\
\left.K_{i} \nabla u_{i} \cdot \nu\right|_{\Gamma_{N} \times \mathbf{R}^{+}} & =\gamma_{i} \\
\left.u_{i}\right|_{\Gamma_{D} \times \mathbf{R}^{+}} & =g_{i} \\
{\left[K_{i} \nabla u_{i} \cdot \nu\right]_{\Gamma_{i n t} \times \mathbf{R}^{+}}} & =\varrho_{i} \\
{\left[u_{i}\right]_{\Gamma_{i n t} \times \mathbf{R}^{+}}} & =0 \\
\left.u_{i}\right|_{t=0} & =u_{i}^{(0)}
\end{array}\right\}
$$

where $t \geq 0$ is the time variable and $x$ is the space variable in a $d$-dimensional bounded domain $\Omega$ (where $d=1,2$ or 3). A precise formulation of the conditions and notations of the problem will be given in the next section. Such coupled nonlinearities frequently arise in environmental modeling, in particular, in the study of the transport of air pollutants, where $u_{i}$ are concentrations of chemical species. These systems may consist of a huge number of equations, e.g. in [15] a model with more than 70 chemical reaction is analysed in details, but larger models can also occur. We have considered such problems with only Dirichlet boundary conditions and without interface conditions in [11]. Thereby a compound algorithm was defined and its efficiency was demonstrated by numerical tests.

Our goal is to extend the approach of [11] to problems with mixed boundary conditions and interface conditions. The mixed boundary conditions allow that the flux is prescribed on a part of the boundary, whereas the presence of interface conditions allows the inclusion of a localized reaction on some subdomain modelled by a curve or polygon. Similarly as in [11], we first apply time-discretization, thus a coupled elliptic system has to be solved on each time level. Since the system of ODEs that stems from the chemical part is generally stiff, the usage of implicit time-discretization schemes is inevitable, hence a nonlinearity appears in the elliptic problems. We propose an outer-inner iteration (outer damped inexact Newton method with inner preconditioned conjugate gradient, CG, method) for solving the FEM discretization of the nonlinear elliptic problems. The main part of this method is preconditioning using the discretization of an $\ell$-tuple of independent scalar elliptic operators as preconditioner. This implies that the preconditioning matrix has a block-diagonal structure, and the auxiliary problems can be solved with a cost proportional to that of a single PDE, in contrast to solving the linearized PDE systems. The advantages of such operator preconditioning is discussed in [2]. In particular, the usage of independent scalar operators as preconditioner allows parallelization of the method, which was illustrated for linear elliptic systems in [12].

We note that when similar problems are handled numerically on computers, a very popular and often used approach in this field involves some kind of splitting technique (for example, the Marchuk-Strang splitting). Thereby the problem is split into proper subproblems. In our method no splitting is used, 
since the role of separation to subproblems is played by the above mentioned preconditioning via parallel scalar operators.

The description of the method will consist of four parts: time discretization, space discretization, outer iteration for the nonlinear systems and inner iteration for the linearized systems. The preconditioning step arises in the last part, and we derive mesh independent superlinear convergence of the preconditioned iteration.

\subsection{The numerical solution process and its convergence}

We consider system (1.1) under the following conditions.

\section{Assumptions 1.2.1.}

(i) (Domain:) the domain $\Omega$ is a bounded polytope in $\mathbf{R}^{d}$ with Lipschitz boundary; $\Gamma_{N}, \Gamma_{D} \subset \partial \Omega$ are disjoint subsets, relatively open with respect to $\partial \Omega$ and with piecewise $C^{1}$ boundary, such that $\partial \Omega=\bar{\Gamma}_{D} \cup \bar{\Gamma}_{N}$, further, the interface $\Gamma_{\text {int }} \subset \Omega$ is a piecewise $C^{1}$ surface lying in $\Omega$. Here $\nu$ is the outward normal vector and $[.]_{\Gamma_{\text {int }}}$ denotes the jump (i.e., the difference of the limits from the two sides of the interface $\Gamma_{i n t}$ ) of a function.

(ii) (Smoothness:) for any $i=1, \ldots, \ell, \quad K_{i} \in L^{\infty}(\Omega), \quad \boldsymbol{b}_{i} \in C^{1}(\bar{\Omega})^{d}, \quad f_{i} \in$ $L^{2}(\Omega), \quad g_{i}$ is the trace of a Dirichlet lift $\tilde{g}_{i} \in H^{1}(\Omega), \gamma_{i} \in L^{2}\left(\Gamma_{N}\right)$ and $\varrho_{i} \in L^{2}\left(\Gamma_{i n t}\right)$. Further, the function $R=\left(R_{1}, \ldots, R_{\ell}\right): \Omega \times \mathbf{R}^{\ell} \rightarrow \mathbf{R}^{\ell}$ is measurable and bounded w.r. to the variable $x \in \Omega$ and $C^{1}$ in the variable $\xi \in \mathbf{R}^{\ell}$.

(iii) (Boundedness from below:) there is $m>0$ such that $K_{i} \geq m$ holds for all $i=1, \ldots, \ell$, further, using the notation $R_{\xi}^{\prime}(x, \xi):=\frac{\partial R(x, \xi)}{\partial \xi}$, there exists $\alpha \in \mathbf{R}$ such that

$$
R_{\xi}^{\prime}(x, \xi) \eta \cdot \eta-\frac{1}{2}\left(\max _{i} \operatorname{div} \boldsymbol{b}_{i}(x)\right)|\eta|^{2} \geq \alpha|\eta|^{2}
$$

for any $(x, \xi) \in \Omega \times \mathbf{R}^{\ell}$ and $\eta \in \mathbf{R}^{\ell}$.

(iv) (Local Lipschitz continuity:) let $3 \leq p$ (if $d=2$ ) or $3 \leq p \leq 6$ (if $d=3)$, then there exist constants $c_{1}, c_{2} \geq 0$ such that for any $\left(x, \xi_{1}\right)$ and $\left(x, \xi_{2}\right) \in \Omega \times \mathbf{R}^{l}$,

$$
\left\|R_{\xi}^{\prime}\left(x, \xi_{1}\right)-R_{\xi}^{\prime}\left(x, \xi_{2}\right)\right\| \leq\left(c_{1}+c_{2}\left(\max \left|\xi_{1}\right|,\left|\xi_{2}\right|\right)^{p-3}\right)\left|\xi_{1}-\xi_{2}\right| .
$$

First let us formulate our problem using a vector notation, i.e. we set for brevity

$$
\boldsymbol{u}:=\boldsymbol{u}(x, t)=\left(u_{1}(x, t), u_{2}(x, t), \ldots, u_{\ell}(x, t)\right),
$$

which satisfies the semilinear parabolic system 


$$
\begin{aligned}
\frac{\partial \boldsymbol{u}}{\partial t}-\operatorname{div}(\boldsymbol{K} \nabla \boldsymbol{u})+\boldsymbol{b} \cdot \nabla \boldsymbol{u}+\boldsymbol{R}(x, \boldsymbol{u}) & =\boldsymbol{f}, \\
\left.\boldsymbol{K} \nabla \boldsymbol{u} \cdot \nu\right|_{\Gamma_{N} \times \mathbf{R}^{+}} & =\boldsymbol{\gamma}, \\
\left.\boldsymbol{u}\right|_{\Gamma_{D} \times \mathbf{R}^{+}} & =\boldsymbol{g}, \\
{[\boldsymbol{K} \nabla \boldsymbol{u} \cdot \nu]_{\Gamma_{i n t} \times \mathbf{R}^{+}} } & =\boldsymbol{\varrho}, \\
{[\boldsymbol{u}]_{\Gamma_{i n t} \times \mathbf{R}^{+}} } & =0 .
\end{aligned}
$$

The numerical solution starts by using Röthe's method, see [9], where the PDE system is first discretized in time, in general by a Runge-Kutta method, but in our situation it is proper to use the implicit (backward) Euler method. Then the resulting nonlinear equation will be discretized by finite elements and solved by an iterative method combined with a preconditioning process (as an inner iteration).

\subsubsection{Time discretization}

First we apply time discretization on the continuous level to system (1.3). For stability reasons we apply the implicit Euler method. The convergence for general time discretizations of quasilinear Cauchy problems is found e.g. in the paper [13]. For ease of presentation we use constant step size $\tau$. (We denote $v^{n}:=v(., n \tau)$ for any continuous function.)

This results in the following nonlinear elliptic system on each time level, for $n \geq 1$ :

$$
\begin{aligned}
-\tau \operatorname{div}\left(\boldsymbol{K} \nabla \boldsymbol{u}^{n+1}\right)+\tau \boldsymbol{b} \cdot \nabla \boldsymbol{u}^{n+1}+\tau \boldsymbol{R}\left(x, \boldsymbol{u}^{n+1}\right)+\boldsymbol{u}^{n+1} & =\tau \boldsymbol{f}^{n+1}+\boldsymbol{u}^{n}, \\
\left.\boldsymbol{K} \nabla \boldsymbol{u}^{n+1} \cdot \nu\right|_{\Gamma_{N}} & =\gamma^{n+1}, \\
\left.\boldsymbol{u}^{n+1}\right|_{\Gamma_{D}} & =\boldsymbol{g}^{n+1} \\
{\left[\boldsymbol{K} \nabla \boldsymbol{u}^{n+1} \cdot \nu\right]_{\Gamma_{i n t}} } & =\boldsymbol{\varrho}^{n+1} \\
{\left[\boldsymbol{u}^{n+1}\right]_{\Gamma_{i n t}} } & =0 .
\end{aligned}
$$

\subsubsection{FEM discretization in space}

First, the weak formulation of the problem is done after homogenization of the boundary conditions (i.e. we set $u_{i} \rightarrow u_{i}-\tilde{g}_{i}$, where $\tilde{g}_{i}$ is the Dirichlet lift for $g_{i}$ ). Defining the Sobolev space

$$
H_{D}^{1}(\Omega):=\left\{u \in H^{1}(\Omega): u_{\mid \Gamma_{D}}=0\right\}
$$


that corresponds to the decomposition to $\Gamma_{N}$ and $\Gamma_{D}$, we will use the product space $H_{D}^{1}(\Omega)^{\ell}$ for system (1.1). Thereby we use the following nonlinear operator: for any $\boldsymbol{u} \in H_{D}^{1}(\Omega)^{\ell}$ let

$$
\begin{aligned}
& \langle F(\boldsymbol{u}), \boldsymbol{v}\rangle_{H_{D}^{1}(\Omega)^{\ell}}=\int_{\Omega} \sum_{i=1}^{\ell}\left(\tau K_{i} \nabla u_{i}^{n+1} \cdot \nabla v_{i}+\tau\left(\mathbf{b}_{i} \cdot \nabla u_{i}^{n+1}\right) v_{i}\right. \\
& \left.+\tau R_{i}\left(x, \boldsymbol{u}^{n+1}\right) v_{i}+u_{i}^{n+1} v_{i}\right) \\
& =\int_{\Omega}\left(\tau \boldsymbol{K} \nabla \boldsymbol{u}^{n+1} \cdot \nabla \boldsymbol{v}+\tau\left(\mathbf{b} \cdot \nabla \boldsymbol{u}^{n+1}\right) \cdot \boldsymbol{v}\right. \\
& \left.+\tau \boldsymbol{R}\left(x, \boldsymbol{u}^{n+1}\right) \cdot \boldsymbol{v}+\boldsymbol{u}^{n+1} \cdot \boldsymbol{v}\right) \quad\left(\boldsymbol{v} \in H_{D}^{1}(\Omega)^{\ell}\right) .
\end{aligned}
$$

The weak form of the right hand side can be represented as follows, where for simplicity we define

$$
\Gamma:=\Gamma_{N} \cup \Gamma_{\text {int }},
$$

and we extend the functions $\gamma_{i}$ and $\varrho_{i}$ to $\Gamma$ as constant zero on the other component. Thus for all $\boldsymbol{v} \in H_{D}^{1}(\Omega)^{\ell}$ we let

$$
\begin{aligned}
\langle B, \boldsymbol{v}\rangle_{H_{D}^{1}(\Omega)^{\ell}} & =\int_{\Omega} \sum_{i=1}^{\ell}\left(\tau f_{i}^{n+1} v_{i}+u_{i}^{n} v_{i}\right) \mathrm{d} x+\tau \int_{\Gamma} \sum_{i=1}^{\ell}\left(\gamma_{i} v_{i}+\varrho_{i} v_{i}\right) \mathrm{d} \sigma \\
& =\int_{\Omega}\left(\tau \boldsymbol{f}^{n+1} \cdot \boldsymbol{v}+\boldsymbol{u}^{n} \cdot \boldsymbol{v}\right)+\tau \int_{\Gamma} \sum_{i=1}^{\ell}(\boldsymbol{\gamma} \cdot \boldsymbol{v}+\boldsymbol{\varrho} \cdot \boldsymbol{v}) \mathrm{d} \sigma .
\end{aligned}
$$

Therefore (1.4) is equivalent to the following abstract operator equation:

$$
\langle F(\boldsymbol{u}), \boldsymbol{v}\rangle_{H_{D}^{1}(\Omega)^{\ell}}=\langle B, \boldsymbol{v}\rangle_{H_{D}^{1}(\Omega)^{\ell}} \quad\left(\boldsymbol{v} \in H_{D}^{1}(\Omega)^{\ell}\right)
$$

We consider the FEM discretization of (1.6) over a quasi-uniform triangulation of the domain $\Omega$, i.e. regular triangular finite elements are used in the two-dimensional case, while tetrahedral finite elements are needed in the three-dimensional case. The maximal meshwidth is denoted by $h$. The corresponding finite element space $V_{h} \subset V=H_{D}^{1}(\Omega)$ is spanned by continuous, piecewise linear basis functions $\varphi_{1}, \varphi_{1}, \ldots, \varphi_{N}$ that are continuous on $\Omega$ and linear on each finite element, vanising on $\Gamma_{D}$, and $\varphi_{j}\left(x_{k}\right)=\delta_{j k}$ holds for each node $x_{k}$ (not sitting on $\Gamma_{D}$ ). Then the finite element solution to (1.6) can be simply writen as $\boldsymbol{u}_{h} \in V_{h}^{\ell}$ satisfying

$$
\left\langle F\left(\boldsymbol{u}_{h}\right), \boldsymbol{v}_{h}\right\rangle_{H_{D}^{1}(\Omega)^{\ell}}=\left\langle B, \boldsymbol{v}_{h}\right\rangle_{H_{D}^{1}(\Omega)^{\ell}} \quad\left(\boldsymbol{v}_{h} \in V_{h}^{\ell}\right) .
$$

The convergence of FEM discretization is well-known, see e.g. [4]. 


\subsubsection{Outer iteration: damped Newton's method}

The operator $F_{h}: V_{h}^{\ell} \rightarrow V_{h}^{\ell}$ and the function $\mathbf{g}_{h} \in V_{h}^{\ell}$ are defined by the identities

$$
\begin{aligned}
\left\langle F_{h}\left(\boldsymbol{u}_{h}\right), \boldsymbol{v}_{h}\right\rangle_{H_{0}^{1}} & =\left\langle F\left(\boldsymbol{u}_{h}\right), \boldsymbol{v}_{h}\right\rangle_{H_{0}^{1}} \quad\left(\boldsymbol{v}_{h} \in V_{h}^{\ell}\right), \\
\left\langle\mathbf{g}_{h}, \boldsymbol{v}_{h}\right\rangle_{H_{0}^{1}} & =\int_{\Omega} \mathbf{g} \cdot \boldsymbol{v}_{h} \quad\left(\boldsymbol{v}_{h} \in V_{h}^{\ell}\right)
\end{aligned}
$$

via the Riesz representation theorem, thus the problem can be written as a nonlinear algebraic system

$$
F_{h}\left(\boldsymbol{u}_{h}\right)=\mathbf{g}_{h}
$$

We apply the damped inexact Newton method (DIN) for the iterative solution of problem (1.7). The construction of the DIN method and the related convergence results are well-known, for completeness we briefly summarize them as follows.

Algorithm 1.2.1 (DIN) Let $\boldsymbol{u}_{0} \in V_{h}^{\ell}$ be arbitrary. The sequence $\left(\boldsymbol{u}_{n}\right) \subset$ $V_{h}^{\ell}$ is constructed as follows:

- Denoting the residual by $\mathbf{r}_{h}=\mathbf{g}_{h}-F_{h}\left(\boldsymbol{u}_{n}\right)$, the vector $\mathbf{p}_{n}$ is the approximate solution of problem $F_{h}^{\prime}\left(\boldsymbol{u}_{n}\right) \mathbf{p}_{n}=\mathbf{r}_{\mathbf{h}}$, i.e.

$$
\left\|F_{h}^{\prime}\left(\boldsymbol{u}_{n}\right) \mathbf{p}_{n}-\mathbf{r}_{\mathbf{h}}\right\|_{H_{0}^{1}} \leq \delta_{n}\left\|\mathbf{r}_{h}\right\|_{H_{0}^{1}} \quad \text { with } 0<\delta_{n} \leq \delta_{0}<1,
$$

- $\sigma_{n}=\min \left\{1, \frac{1-\delta_{n}}{\left(1+\delta_{n}\right)^{2}} \cdot \frac{K^{2}}{L\left\|F_{h}\left(\boldsymbol{u}_{n}\right)-\mathbf{g}_{h}\right\|_{H_{0}^{1}}}\right\}$,

- $\boldsymbol{u}_{n+1}=\boldsymbol{u}_{n}+\sigma_{n} \mathbf{p}_{n}$.

Under suitable smoothness, growth and coercivity conditions the following theorem holds, for the proof see [6, Thm. 5.12] in a more general setting.

Theorem 1. If $\delta_{n} \leq c \cdot\left\|F_{h}\left(\boldsymbol{u}_{n}\right)-\mathbf{g}_{h}\right\|_{H_{0}^{1}}^{\gamma}$ with some $0<\gamma \leq 1$, then the convergence is locally of order $1+\gamma$, that is the convergence is linear for $n_{0}$ steps until $\left\|F_{h}\left(\boldsymbol{u}_{n}\right)-\mathbf{g}_{h}\right\|_{H_{0}^{1}}^{\gamma} \leq \epsilon$, where $\epsilon \leq\left(1-\delta_{0}\right) \frac{K^{2}}{2 L}$ (here and in the definition of $\sigma_{n}$ the constant $L$ comes from the Lipschitz continuity of $\left.F^{\prime}\right)$, and further on (as $\sigma_{n} \equiv 1$ )

$$
\left\|\boldsymbol{u}_{n}-\boldsymbol{u}_{h}\right\|_{H_{0}^{1}} \leq d_{1} q^{(1+\gamma)^{n-n_{0}}}
$$

with some $d_{1}>0,0<q<1$. 


\subsubsection{Inner iteration: preconditioned CG method using equivalent operator preconditioning}

In each step the construction of $\boldsymbol{u}_{n}$ requires the approximate solution of the linearized problem

$$
F_{h}^{\prime}\left(\boldsymbol{u}_{n}\right) \mathbf{p}_{h}=\mathbf{r}_{h}
$$

Applying the previously mentioned homogenization and linearization, and dividing by $\tau$, it is easy to see that the linearized system is equivalent to the FEM solution in $V_{h}^{\ell}$ of a linear elliptic system of the following structure:

$$
\left.\begin{array}{rl}
-\operatorname{div}\left(K_{i} \nabla p_{i}\right)+\mathbf{b}_{i} \cdot \nabla p_{i}+\sum_{j=1}^{\ell} \hat{R}_{i j} p_{j} & =\hat{f}_{i} \\
\left.K_{i} \nabla p_{i} \cdot \nu\right|_{\Gamma_{N}} & =\hat{\gamma}_{i} \\
\left.p_{i}\right|_{\Gamma_{D}} & =0 \\
{\left[K_{i} \nabla p_{i} \cdot \nu\right]_{\Gamma_{i n t}}} & =\hat{\varrho}_{i} \\
{\left[p_{i}\right]_{\Gamma_{i n t}}} & =0
\end{array}\right\}
$$

where the coefficients of the $p_{j}$ are

$$
\hat{R}_{i j}(x):=\partial_{j} R_{i}\left(x, \boldsymbol{u}_{n}(x)+\tilde{g}_{i}(x)\right)+\frac{1}{\tau} \delta_{i j}
$$

(where $\delta_{i j}$ denotes the Kronecker symbol). The weak form of problem (1.9) on the continuous level is

$$
\begin{aligned}
& \int_{\Omega} \sum_{i=1}^{\ell}\left(K_{i} \nabla p_{i} \cdot \nabla v_{i}+\left(\mathbf{b}_{i} \cdot \nabla p_{i}\right) v_{i}+\sum_{j=1}^{\ell} \hat{R}_{i j} p_{j} v_{i}\right) \\
= & \int_{\Omega} \sum_{i=1}^{\ell} \hat{f}_{i} v_{i} \mathrm{~d} x+\int_{\Gamma} \sum_{i=1}^{\ell}\left(\hat{\gamma}_{i} v_{i}+\hat{\varrho}_{i} v_{i}\right) \mathrm{d} \sigma \quad\left(\boldsymbol{v}_{h} \in V_{h}^{\ell}\right) .
\end{aligned}
$$

Denoting by $\mathbf{c}$ and $\mathbf{d}$ the coefficient vectors of $\mathbf{p}_{h}$ and $\mathbf{r}_{h}$ with respect to the FEM basis $\left\{\phi_{1}, \ldots, \phi_{N}\right\}$, and by $\mathbf{L}_{h}^{(n)}$ the stiffness matrix corresponding to the left-hand side of (1.9), Eq. (1.8) turns into the linear algebraic system

$$
\mathbf{L}_{h}^{(n)} \mathbf{c}=\mathbf{d} .
$$

The theory of equivalent operators (cf. [2]) can be applied to the auxiliary linear problem (1.9) which can be solved by a proper CG type method using a suitable preconditioner. We propose the CGN method for the nonsymmetric system (1.9), see e.g. [2]. Letting $\eta_{i} \in L^{\infty}(\Omega), \eta_{i} \geq 0$ be suitable functions, we introduce the symmetric linear elliptic operators 


$$
S_{i} p_{i}:=-\operatorname{div}\left(K_{i} \nabla p_{i}\right)+\eta_{i} p_{i} \quad(i=1, \ldots, \ell)
$$

in $H_{D}^{1}(\Omega)$, and then define the $\ell$-tuple of independent elliptic operators

$$
S \mathbf{p}=\left(S_{1} p_{1} S_{2} p_{2} \ldots S_{\ell} p_{\ell}\right)
$$

as preconditioning operator. The preconditioning matrix for the discrete system (1.11) is defined as the stiffness matrix $\mathbf{S}_{h}$ of $S$ in $H_{0}^{1}(\Omega)^{\ell}$. Then we apply the CGN algorithm for the preconditioned system

$$
\mathbf{S}_{h}^{-1} \mathbf{L}_{h}^{(n)} \mathbf{c}=\mathbf{S}_{h}^{-1} \mathbf{d}
$$

where $\tilde{\mathbf{d}}:=\mathbf{S}_{h}^{-1} \mathbf{d}$. According to the main idea of preconditioning, system (1.14) is equivalent to (1.11), but the spectrum of the matrix $\mathbf{S}_{h}^{-1} \mathbf{L}_{h}^{(n)}$ is much more clustered than the spectrum of $\mathbf{L}_{h}^{(n)}$. Therefore, when we apply the CGN algorithm for system (1.14), we obtain fast convergence, which will be analyized below. Altogether, in each Newton step the linearized system (1.9) is preconditioned by the discretization of independent (i.e. decoupled) symmetric scalar elliptic operators. This means that the preconditioning matrix $\mathbf{S}_{h}$ has a block-diagonal structure. This enables parallel computation of the solution of the auxiliary problems in the CGN, which was demonstrated for a linear elliptic test system in [12]. Moreover, combining the convergence results for the CGN and the DIN algorithm 1.2.1, the combined iteration provides mesh independent convergence, with superlinear convergence rate for both the inner and outer iterations. This was given in Theorem 1 for the outer iteration, and it can be derived as follows for the inner iteration.

Theorem 2. (1) The left-hand side of Eq. (1.10) can be represented as

$$
\int_{\Omega} \sum_{i=1}^{\ell}\left(K_{i} \nabla p_{i} \cdot \nabla v_{i}+\left(\mathbf{b}_{i} \cdot \nabla p_{i}\right) v_{i}+\sum_{j=1}^{\ell} \hat{R}_{i j} p_{j} v_{i}\right)=\langle(I+Q) \boldsymbol{p}, \boldsymbol{v}\rangle_{H_{D}^{1}(\Omega)^{\ell}},
$$

for any $\boldsymbol{p}, \boldsymbol{v} \in H_{D}^{1}(\Omega)^{\ell}$, where $Q: H_{D}^{1}(\Omega)^{\ell} \rightarrow H_{D}^{1}(\Omega)^{\ell}$ is a compact linear operator and $I$ is the identity on $H_{D}^{1}(\Omega)^{\ell}$.

(2) The right-hand side of Eq. (1.10) can be represented as

$$
\int_{\Omega} \sum_{i=1}^{\ell} \hat{f}_{i} v_{i} \mathrm{~d} x+\int_{\Gamma} \sum_{i=1}^{\ell}\left(\hat{\gamma}_{i} v_{i}+\hat{\varrho}_{i} v_{i}\right) \mathrm{d} \sigma=\langle\boldsymbol{r}, \boldsymbol{v}\rangle_{H_{D}^{1}(\Omega)^{\ell}} \quad\left(\boldsymbol{v} \in H_{D}^{1}(\Omega)^{\ell}\right)
$$

for a proper element $\boldsymbol{r} \in H_{D}^{1}(\Omega)^{\ell}$.

Proof. (1) Let us introduce the weighted inner product

$$
\langle\boldsymbol{u}, \boldsymbol{v}\rangle_{H_{D}^{1}(\Omega)^{\ell}}:=\int_{\Omega} \sum_{i=1}^{\ell}\left(K_{i} \nabla u_{i} \cdot \nabla v_{i}+\eta_{i} u_{i} v_{i}\right)
$$


in the space $H_{D}^{1}(\Omega)^{\ell}$, which is equivalent to the standard one. Defining $Q$ via

$$
\langle Q \boldsymbol{p}, \boldsymbol{v}\rangle_{H_{D}^{1}(\Omega)^{\ell}}:=\int_{\Omega} \sum_{i=1}^{\ell}\left(\left(\mathbf{b}_{i} \cdot \nabla p_{i}\right) v_{i}+\sum_{j=1}^{\ell} \hat{R}_{i j} p_{j} v_{i}-\eta_{i} p_{i} v_{i}\right) \quad\left(\boldsymbol{p}, \boldsymbol{v} \in H_{D}^{1}(\Omega)^{\ell}\right),
$$

the representation is obvious under the used weighted inner product. The compactness of $Q$ follows from the fact that it is the sum of the weak forms of first and zeroth order scalar operators, for which the compactness was shown under the similar form in [3].

(2) The continuous embedding of $L^{2}(\Omega)$ and $L^{2}(\Gamma)$ into $H_{D}^{1}(\Omega)$ implies that each term in the right-hand side of (1.10) is a bounded linear functional on $H_{D}^{1}(\Omega)$ w.r.t the variable $v_{i}$. Hence the sum of these terms is a bounded linear functional on $H_{D}^{1}(\Omega)^{\ell}$ w.r.t the variable $\boldsymbol{v}$. Then the Riesz representation theorem yields the existence and uniqueness of the proper element $\boldsymbol{r} \in H_{D}^{1}(\Omega)^{\ell}$.

Theorem 3. Let us apply the CGN algorithm for system (1.14) under the $\mathbf{S}_{h}$-inner product $\langle., .\rangle_{\mathbf{S}_{h}}$. Then the residual errors satisfy

$$
\left(\frac{\left\|r_{k}\right\|_{\mathbf{S}_{h}}}{\left\|r_{0}\right\|_{\mathbf{S}_{h}}}\right)^{1 / k} \leq \varepsilon_{k}
$$

where

$$
\varepsilon_{k}:=\frac{2}{k m^{2}} \sum_{i=1}^{k}\left(\left|\lambda_{i}\left(Q^{*}+Q\right)\right|+\lambda_{i}\left(Q^{*} Q\right)\right) \rightarrow 0 \quad \text { as } k \rightarrow \infty
$$

(with $Q$ being the compact operator above, and $m$ being the ellipticity constant of $I+Q)$, and $\varepsilon_{k}$ is a sequence independent of $V_{h}$.

Proof. Due to Theorem 3, we can rewrite Eq. (1.10) as

$$
(I+Q) \boldsymbol{p}=\boldsymbol{r}
$$

in $H_{D}^{1}(\Omega)^{\ell}$, i.e. an operator equation with a compact preturbation of the identity. Similarly, since we have the decomposition

$$
\mathbf{L}_{h}^{(n)}=\mathbf{S}_{h}+\mathbf{Q}_{h}^{(n)}
$$

(where respectively $\mathbf{S}_{h}^{(n)}$ and $\mathbf{Q}_{h}^{(n)}$ are the stiffness matrices for the weighted inner product and compact operator $Q$, defined in Theorem 3), we obtain that (1.14) can be rewritten as

$$
\left(\mathbf{I}_{h}+\mathbf{S}_{h}^{-1} \mathbf{Q}_{h}^{(n)}\right) \mathbf{c}=\widetilde{\mathbf{d}}
$$


(where $\widetilde{\mathbf{d}}:=\mathbf{S}_{h}^{-1} \mathbf{d}$ ). That is, we obtain a proper projection of (1.17) in $V_{h}^{\ell}$, and Proposition 7.5 of [2] yields the desired convergence result.

\subsection{Some examples in air pollution models}

Systems of the type (1.1) typically arise in air pollution models, involving diffusion, convection and chemical reactions related to the polluting materials (pollutants), see e.g. [14, 15]. A simplified model involving the 10 main arising pollutants involves the following vector, describing the concentrations of the considered species:

$$
\boldsymbol{u}=\left(u_{\mathrm{NO}_{2}}, u_{\mathrm{O}_{3}}, u_{\mathrm{NO}}, u_{\mathrm{O}_{2}}, u_{\mathrm{O}^{1} \mathrm{D}}, u_{\mathrm{H}_{2} \mathrm{O}}, u_{\mathrm{OH}}, u_{\mathrm{CO}}, u_{\mathrm{H}}, u_{\mathrm{HO}_{2}}\right)^{T} .
$$

Then the reaction terms, given through the coordinate functions of $R$, are as follows:

$$
\begin{aligned}
R_{\mathrm{NO}_{2}}(\boldsymbol{u})= & -J_{1} u_{\mathrm{NO}_{2}} u_{h v}+k_{2} u_{\mathrm{NO}} u_{\mathrm{O}_{3}}-k_{5} u_{\mathrm{OH}} u_{\mathrm{NO}_{2}}+k_{8} u_{\mathrm{HO}_{2}} u_{\mathrm{NO}} \\
R_{\mathrm{O}_{3}}(\boldsymbol{u})= & J_{1} u_{\mathrm{NO}_{2}} u_{h v}-k_{2} u_{\mathrm{NO}} u_{\mathrm{O}_{3}}-J_{3} u_{\mathrm{O}_{3}} u_{h v}+k_{9}(T) u_{\mathrm{O}^{1} \mathrm{D}} u_{M} \\
R_{\mathrm{NO}}(\boldsymbol{u})= & J_{1} u_{\mathrm{NO}_{2}} u_{h v}-k_{2} u_{\mathrm{NO}} u_{\mathrm{O}_{3}}-k_{8} u_{\mathrm{HO}_{2}} u_{\mathrm{NO}} \\
R_{\mathrm{O}_{2}}(\boldsymbol{u})= & k_{2} u_{\mathrm{NO}} u_{\mathrm{O}_{3}}+J_{3} u_{\mathrm{O}_{3}} u_{h v} \\
R_{\mathrm{O}^{1} \mathrm{D}}(\boldsymbol{u})= & J_{3} u_{\mathrm{O}_{3}} u_{h v}-k_{4} u_{\mathrm{O}^{1} \mathrm{D}} u_{\mathrm{H}_{2} \mathrm{O}}-k_{9}(T) u_{\mathrm{O}^{1} \mathrm{D}} u_{M} \\
R_{\mathrm{H}_{2} \mathrm{O}}(\boldsymbol{u})= & -k_{4} u_{\mathrm{O}^{1} \mathrm{D}} u_{\mathrm{H}_{2} \mathrm{O}}+k_{10} u_{H C H O} u_{\mathrm{OH}} \\
R_{\mathrm{OH}}(\boldsymbol{u})= & 2 k_{4} u_{\mathrm{O}^{1} \mathrm{D}} u_{\mathrm{H}_{2} \mathrm{O}}-k_{5} u_{\mathrm{OH}} u_{\mathrm{NO}_{2}}-k_{6} u_{\mathrm{OH}} u_{\mathrm{CO}}+k_{8} u_{\mathrm{HO}_{2}} u_{\mathrm{NO}} \\
& -k_{10} u_{H C H O} u_{\mathrm{OH}} \\
R_{\mathrm{CO}}(\boldsymbol{u})= & -k_{6} u_{\mathrm{OH}} u_{\mathrm{CO}}+k_{10} u_{H C H O} u_{\mathrm{OH}}+J_{11} u_{H C H O} u_{h v} \\
& +J_{12} u_{H C H O} u_{h v} \\
R_{\mathrm{H}}(\boldsymbol{u})= & k_{6} u_{\mathrm{OH}} u_{\mathrm{CO}}-k_{7}(T) u_{\mathrm{H}} u_{\mathrm{O}_{2}} u_{M} \\
R_{\mathrm{HO}_{2}}(\boldsymbol{u})= & k_{7}(T) u_{\mathrm{H}} u_{\mathrm{O}_{2}} u_{M}-k_{8} u_{\mathrm{HO}_{2}} u_{\mathrm{NO}}+k_{10} u_{H C H O} u_{\mathrm{OH}} \\
& +2 J_{11} u_{H C H O} u_{h v} .
\end{aligned}
$$

Here $k_{j}$ are the reaction rates, and the photolysis rates are defined as

$$
J_{j}=a_{j} e^{-b_{j} \sin (\theta)^{-1}} .
$$

Let us list some possible situations covered by our system (1.1). This shows that our given numerical algorithm can be used for the computation of the desired concentrations. The initial conditions have the same general form

$$
\left.u_{i}\right|_{t=0}=u_{i}^{(0)}
$$

as in (1.1) in each problem below, hence it is omitted in the formulas. 
Example 1.3.1. Dirichlet boundary conditions in 2D:

$$
\left.\begin{array}{rl}
\frac{\partial u_{i}}{\partial t}-\operatorname{div}\left(K_{i} \nabla u_{i}\right)+\mathbf{b}_{i} \cdot \nabla u_{i}+R_{i}\left(x, u_{1}, \ldots, u_{\ell}\right) & =f_{i} \\
\left.u_{i}\right|_{\partial \Omega \times \mathbf{R}^{+}} & =g_{i}
\end{array}\right\} \quad(i=1, \ldots, \ell) .
$$

In this case the values of concentrations are given from a larger-scale model on the boundary of the considered, typically rectangular domain. The exact boundary conditions are of course unknown in real situations, therefore these values should be taken from a model treated on a much larger spatial domain. Some kind of interpolation may be needed because the larger models are normally handled on the coarser grids, see details in [15]. This model was studied in [11], and the efficiency of the similar approach was demonstrated by numerical test, involving a rotating wind field test problem taken from [7].

Example 1.3.2. Mixed boundary conditions in 3D:

$$
\left.\begin{array}{rl}
\frac{\partial u_{i}}{\partial t}-\operatorname{div}\left(K_{i} \nabla u_{i}\right)+\mathbf{b}_{i} \cdot \nabla u_{i}+R_{i}\left(x, u_{1}, \ldots, u_{\ell}\right) & =f_{i} \\
\left.K_{i} \nabla u_{i} \cdot \nu\right|_{\Gamma_{N} \times \mathbf{R}^{+}} & =0 \\
\left.u_{i}\right|_{\Gamma_{D} \times \mathbf{R}^{+}} & =g_{i}
\end{array}\right\} \quad(i=1, \ldots, \ell)
$$

Here $\Omega$ is a 3D rectangle, $\Gamma_{N}$ is the union of the horizontal faces of the domain and there the flux is assumed to be zero, i.e. no horizontal tramsmission occurs, whereas $\Gamma_{D}$ has the same role as in the previous example.

Example 1.3.3. Mixed boundary conditions in 2D, point source:

$$
\left.\begin{array}{rl}
\frac{\partial u_{i}}{\partial t}-\operatorname{div}\left(K_{i} \nabla u_{i}\right)+\mathbf{b}_{i} \cdot \nabla u_{i}+R_{i}\left(x, u_{1}, \ldots, u_{\ell}\right) & =f_{i} \\
\left.K_{i} \nabla u_{i} \cdot \nu\right|_{\Gamma_{N} \times \mathbf{R}^{+}} & =\gamma_{i} \\
\left.u_{i}\right|_{\Gamma_{D} \times \mathbf{R}^{+}} & =g_{i}
\end{array}\right\} \quad(i=1, \ldots, \ell) .
$$

Here $\Gamma_{D}$ is a small circle inside $\Omega$ representing a chimney which is a(n almost) point source of the pollution, $g_{i}$ are given large values, and $\Gamma_{N}$ is the outer boundary where the flux is given.

Example 1.3.4. Dirichlet boundary conditions in 2D and interface conditions on a curve: 


$$
\left.\begin{array}{rl}
\frac{\partial u_{i}}{\partial t}-\operatorname{div}\left(K_{i} \nabla u_{i}\right)+\mathbf{b}_{i} \cdot \nabla u_{i}+R_{i}\left(x, u_{1}, \ldots, u_{\ell}\right) & =f_{i} \\
\left.u_{i}\right|_{\partial \Omega \times \mathbf{R}^{+}} & =g_{i} \\
{\left[K_{i} \nabla u_{i} \cdot \nu\right]_{\Gamma_{i n t} \times \mathbf{R}^{+}}} & =\varrho_{i} \\
{\left[u_{i}\right]_{\Gamma_{i n t} \times \mathbf{R}^{+}}} & =0
\end{array}\right\}
$$

Here Example 1 is completed such that the diffusion coefficient is different on the two sides of an interior curve $\Gamma_{i n t}$ that separates two subregions, e.g. the boundary of a lake.

In addition, our results might be extended to include reaction terms also on the interface $\Gamma_{i n t}$, which we did not do for simplicity. Then one can consider problems like

$$
\left.\begin{array}{rl}
\frac{\partial u_{i}}{\partial t}-\operatorname{div}\left(K_{i} \nabla u_{i}\right)+\mathbf{b}_{i} \cdot \nabla u_{i}+R_{i}\left(x, u_{1}, \ldots, u_{\ell}\right) & =f_{i} \\
\left.u_{i}\right|_{\partial \Omega \times \mathbf{R}^{+}} & =g_{i} \\
{\left[K_{i} \nabla u_{i} \cdot \nu+s_{i}\left(x, u_{1}, \ldots, u_{\ell}\right)\right]_{\Gamma_{i n t} \times \mathbf{R}^{+}}} & =\varrho_{i} \\
{\left[u_{i}\right]_{\Gamma_{i n t} \times \mathbf{R}^{+}}} & =0
\end{array}\right\}
$$

in which given chemical reactions, described by functions $s_{i}$, take place on the interior curve as considered e.g. in [10]. Such a curve can model a localized site of catalysis, or a polluted highway etc. The formal conditions for the functions $s_{i}$ are similar as for $R_{i}$.

Finally we note that it is not the goal of this paper to include computer tests, since we have focused on the general approach to construct methods for a class of problems. It will be the subject of forthcoming papers to implement such algorithms for specific problems like in the above examples. We expect as similarly efficient behaviour for such problems as found in the tests for the pure Dirichlet problem in our cited paper [11].

Acknowledgement. This research was supported by the by the Hungarian Scientific Research Fund OTKA, No. 112157.

\section{References}

1. Axelsson, O., Iterative Solution Methods, Cambridge University Press, 1994.

2. Axelsson, O., Karátson J., Equivalent operator preconditioning for elliptic problems. Numer. Algor., 50:297-380, 2009.

3. Axelsson, O., Karátson J., Mesh independent superlinear PCG rates via compactequivalent operators, SIAM J. Numer. Anal., 45 (2007), No.4, pp. 1495-1516

4. Ciarlet, P.G. The finite element method for elliptic problems SIAM, 2002.

5. Elman, H. C., Silvester, D. J., Wathen, A. J., Finite Elements and Fast Iterative Solvers: with Applications in Incompressible Fluid Dynamics, Numerical Mathematics and Scientific Computation, Oxford University Press, New York, 2005. 
6. Faragó, I., Karátson, J., Numerical solution of nonlinear elliptic problems via preconditioning operators. Theory and applications. Advances in Computation, Volume 11, NOVA Science Publishers, New York, 2002.

7. Georgiev, K., Zlatev, Z., Implementation of sparse matrix algorithms in an advection-diffusion-chemistry module. J. Comput. Appl. Math., 236(3):342-353, 2011.

8. Hov, Ø., Zlatev, Z., Berkowicz, R., Eliassen, A., Prahm, L. P., Comparison of numerical techniques for use in air pollution models with non-linear chemical reactions, Atmospheric Environment, 23(5):967-983, 1989.

9. KACUR, J., Method of Rothe in Evolution Equations, Teubner-Texte zur Mathematik, Bd. 80, Leipzig (1985).

10. Kandilarov, J. D., Vulkov, L. G., Analysis of immersed interface difference schemes for reaction-diffusion problems with singular own sources, Comput. Methods Appl. Math. 3 (2003), no. 2, 253-273.

11. KARÁtson J., Kurics T., A preconditioned iterative solution scheme for nonlinear parabolic systems arising in air pollution modeling, Mathematical Modelling and Analysis 18(5) (2013), pp. 641653.

12. Karátson J., Kurics T., Lirkov, I., A Parallel Algorithm for Systems of ConvectionDiffusion Equations, in: NMA 2006, eds. T. Boyanov et al., Lecture Notes Comp. Sci. 4310, pp. 65-73, Springer, 2007.

13. Lubich, Ch., Ostermann, A., Runge-Kutta approximation of quasilinear parabolic equations, Math. Comp. 64, 601-627 (1995).

14. Tomlin, A., Berzins, M., Ware, J., Smith, J., Pilling, M.J., On the use of adaptive gridding methods for modelling chemical transport from multi-scale sources, Atmospheric Emironment, Vol. 31, No. 18. pp. 2945-2959, 1997.

15. Zlatev, Z., Computer treatment of large air pollution models, Kluwer Academic Publishers, Dordrecht-Boston-London, 1995

16. Zlatev, Z., Dimov, I., Computational and Numerical Challenges in Air Pollution Modelling, Studies in Computational Mathematics. Elsevier Science, 2006. 